\title{
A LARGE DEVIATIONS PERSPECTIVE ON ORDINAL OPTIMIZATION
}

\author{
Peter Glynn \\ Department of Management Science and Engineering \\ Stanford University \\ Stanford, CA 94305, U.S.A.
}

\author{
Sandeep Juneja \\ School of Technology and Computer Science \\ Tata Institute of Fundamental Research \\ Mumbai, INDIA - 400005
}

\begin{abstract}
We consider the problem of optimal allocation of computing budget to maximize the probability of correct selection in the ordinal optimization setting. This problem has been studied in the literature in an approximate mathematical framework under the assumption that the underlying random variables have a Gaussian distribution. We use the large deviations theory to develop a mathematically rigorous framework for determining the optimal allocation of computing resources even when the underlying variables have general, non-Gaussian distributions. Further, in a simple setting we show that when there exists an indifference zone, quick stopping rules may be developed that exploit the exponential decay rates of the probability of false selection. In practice, the distributions of the underlying variables are estimated from generated samples leading to performance degradation due to estimation errors. On a positive note, we show that the corresponding estimates of optimal allocations converge to their true values as the number of samples used for estimation increases to infinity.
\end{abstract}

\section{INTRODUCTION}

Suppose that we have a set of $d$ populations and we can sample independently from each using simulation. Let $X_{i}$ be a random variable sampled from population $i$ and let $\mu_{i}=E X_{i}$ denote its expectation. Further suppose that the parameters $\left(\mu_{i}: i \leq d\right)$ are unknown. We consider the problem of correctly selecting the population with the smallest mean based on the samples observed from each. Applications of this arise in selecting the best design from a set of competing designs, all of which may be modeled as discrete event dynamic systems. The selection of the best design is based on a performance measure that may be represented as an expectation of a random variable whose samples can be generated via simulation, although analytically evaluating the expectation may be an intractable problem.
One approach to tackle this problem is to use simulation to estimate each $\mu_{i}$ accurately and then compare the respective values of the estimates. Since the rate of convergence of the simulated estimate is $O(1 / \sqrt{n})$, where $n$ is the number of samples generated (and hence is a measure of computational effort), the amount of computational effort needed to accurately estimate each mean can be very large. The ordinal optimization involves generating samples from each population and selecting the one with the lowest sample mean. In Dai (1996), it is shown that using ordinal optimization the probability of correct selection converges at an exponential rate for a large class of systems.

In this paper we consider the problem of allocating total sampling budget $n$ amongst the $d$ populations in an asymptotically optimal manner (i.e., as $n \rightarrow \infty$ ) so that the probability of correct selection using ordinal optimization is maximized, or equivalently, the probability of false selection is minimized. This problem of optimal allocation has been studied earlier (see Chen et al. 2000) by optimizing an approximation to the probability of correct selection under the assumption that each $X_{i}$ has a Gaussian distribution. In this paper, we allow $X_{i},(1 \leq i \leq d)$ to have a general distribution. We identify the large deviations rate function associated with the random vector of sample means from the $d$ populations, for every budget allocation strategy (see Dembo and Zeitouni 1992 for an introduction to large deviations theory). The problem of optimal allocation that minimizes the probability of false selection then reduces to the problem of finding the allocation that maximizes the rate function over the set of outcomes where false selection is made. We show that this involves solving a simple concave programming problem where the first order conditions are sufficient for optimality. In particular, we show that under the optimal allocation strategy, the probability of falsely selecting any of the competing designs are approximately equal (they are equal on a logarithmic scale).

Suppose that each $X_{i}$ has a non-Gaussian distribution, and to compensate for this, batches of independent samples of each population are considered as a single sample. We note that this amounts to scaling by same amount the rate 
function corresponding to the probability of false selection, under every allocation strategy. Therefore, the optimal allocation is unaffected by the use of batches. In particular, the Gaussian assumption may give substantially sub-optimal allocations in such cases.

Substantial literature exists on selecting the best system amongst many alternatives using ranking/selection procedures (see Goldsman and Nelson 2001, Kim and Nelson 2003 for an overview). These methods rely critically on the assumption that that each $X_{i}$ has a Gaussian distribution. These approaches also consider the 'indifference-zone formulation' where it is assumed that there exists a known $\delta>0$ such that $\mu_{1} \leq \mu_{j}-\delta$ for $j \geq 2$. Such a $\delta$ is then useful in devising rules for the number of samples needed to control the probability of false selection to pre-specified levels.

We also apply this indifference-zone formulation in the ordinal optimization setting when the underlying random variables are Bernoulli. In this simple setting we devise conservative rules to determine the number of samples needed to control the probability of false selection to pre-specified levels, exploiting the exponential decay rates of the probability of false selection.

Identifying the large deviations rate function associated with the probability of false selection involves knowing the $\log$ moment generating function of each $X_{i}$. In practice this has to be estimated from the generated data leading to degradation in performance due to estimation errors. On the positive note, we show that the estimates of the optimal allocations obtained by using the estimated log-moment generating functions are consistent, i.e., in the limit as $n \rightarrow \infty$ they converge to the correct allocations.

In Section 2 we identify the rate function of the sample means from the $d$ populations under any given allocation strategy. We state the theorem characterizing the optimal allocation of sampling budget in Section 3. In Section 4, we incorporate the indifference zone formulation in a simple Bernoulli setting. Finally, in Section 5 we discuss how the large deviations rate function and the associated optimal allocations may be estimated from generated samples and show that such an estimate is consistent.

\section{RATE FUNCTION OF PROBABILITY OF FALSE SELECTION}

Recall that $X_{i}$ is a random sample from population $i$ for $(1 \leq i \leq d)$ and $\mu_{i}=E X_{i}$. Without loss of generality assume that

$$
\mu_{1}<\mu_{2} \leq \ldots \leq \mu_{d}
$$

Consider an allocation strategy that allocates $p_{i} n$ amount of the total sample budget $n$ to the population $i$, where $p_{i}>0,1 \leq i \leq d$ and $\sum_{i=1}^{d} p_{i}=1$. In this paper, we ignore the minor technicalities associated with $p_{i} n$ not being an integer. Let $\left(X_{i, 1}, X_{i, 2}, \ldots, X_{i, p_{i} n}\right)$ denote the samples from population $i, 1 \leq i \leq d$, and let $\bar{X}_{i}\left(p_{i} n\right)$ denote their sample mean.

Note that under the ordinal optimization technique, false selection may be made if $\bar{X}_{1}\left(p_{1} n\right)$ is not the smallest sample mean, i.e., $\bar{X}_{1}\left(p_{1} n\right) \geq \min _{2 \leq j \leq d} \bar{X}_{j}\left(p_{j} n\right)$. Thus, the probability of false selection or $P(F S)$ equals

$$
P\left(\bar{X}_{1}\left(p_{1} n\right) \geq \min _{2 \leq j \leq d} \bar{X}_{j}\left(p_{j} n\right)\right) .
$$

This may be lower bounded by

$$
\max _{2 \leq j \leq d} P\left(\bar{X}_{1}\left(p_{1} n\right) \geq \bar{X}_{j}\left(p_{j} n\right)\right),
$$

and is upper bounded by

$$
(d-1) \max _{2 \leq j \leq d} P\left(\bar{X}_{1}\left(p_{1} n\right) \geq \bar{X}_{j}\left(p_{j} n\right)\right) .
$$

Therefore, if for $2 \leq j \leq d$,

$$
\lim _{n \rightarrow \infty} \frac{1}{n} \log P\left(\bar{X}_{1}\left(p_{1} n\right) \geq \bar{X}_{j}\left(p_{j} n\right)\right)=-G_{j}\left(p_{1}, p_{j}\right)
$$

for some rate function $G_{j}(\cdot, \cdot)$, then

$$
\lim _{n \rightarrow \infty} \frac{1}{n} \log P(F S)=-\min _{2 \leq j \leq d} G_{j}\left(p_{1}, p_{j}\right) .
$$

We determine $G_{j}\left(p_{1}, p_{j}\right)$ using the Gartner-Ellis Theorem (see Dembo and Zeitouni 1992). Some notation and an assumption is needed before we do this.

\subsection{Notation}

Let $\Lambda_{i}(\cdot)$ denote the log-moment generating function of $X_{i}$ and $I_{i}(\cdot)$ denote the Fenchel-Legendre transform of $\Lambda_{i}$, i.e.,

$$
I_{i}(x)=\sup _{\theta}\left(\theta x-\Lambda_{i}(\theta)\right) .
$$

Let $\mathcal{D}_{\Lambda_{i}}=\left\{\theta \in \mathbb{R}: \Lambda_{i}(\theta)<\infty\right)$ and $\mathcal{F}_{i}=\left\{\Lambda_{i}^{\prime}(\theta): \theta \in\right.$ $\left.\mathcal{D}_{\Lambda_{i}^{o}}\right\}$ (for any set $A$, let $A^{o}$ denote its interior). It is well known that $I_{i}(\cdot)$ is strictly convex and $C^{\infty}$ for $x \in \mathcal{F}_{i}^{o}$, $I_{i}\left(\mu_{i}\right)=0$ and $I_{i}(x) \geq 0$ for all $x \in \mathbb{R}$ for $1 \leq i \leq d$ (see Dembo and Zeitouni 1992). We make the following assumption in our analysis.

Assumption 1 The interval $\left[\mu_{1}, \mu_{d}\right] \subset \cap_{i=1}^{d} \mathcal{F}_{i}^{o}$.

Loosely speaking, this assumption ensures that the sample mean from each population can take any value in the interval $\left[\mu_{1}, \mu_{d}\right]$. In particular, it ensures that $P\left(\bar{X}_{1}\left(p_{1} n\right) \geq \bar{X}_{j}\left(p_{j} n\right)\right)>0$ for $2 \leq j \leq d$. It can be checked that if random variables $\left(X_{i}: 1 \leq i \leq d\right)$ belong to commonly encountered family of distributions such 
as the Normal, Bernoulli, Poisson and Gamma family, then Assumption (1) is satisfied.

2.2 Determining $G_{j}\left(p_{1}, p_{j}\right)$

Now we show that

$$
G_{j}\left(p_{1}, p_{j}\right)=\inf _{x}\left(p_{1} I_{1}(x)+p_{j} I_{j}(x)\right) .
$$

Heuristically speaking,

$$
P\left(\bar{X}_{1}\left(p_{1} n\right) \geq \bar{X}_{j}\left(p_{j} n\right)\right) \approx P\left(\bar{X}_{1}\left(p_{1} n\right) \approx \bar{X}_{j}\left(p_{j} n\right)\right)
$$

and the latter approximately equals

$$
\begin{aligned}
& \int_{x} P\left(\bar{X}_{1}\left(p_{1} n\right) \approx \bar{X}_{j}\left(p_{j} n\right) \approx x\right) d x \\
\approx & \int_{x} P\left(\bar{X}_{1}\left(p_{1} n\right) \approx x\right) P\left(\bar{X}_{j}\left(p_{j} n\right) \approx x\right) d x \\
\approx & \int_{x} \exp \left[-p_{1} n I_{1}(x)\right] \exp \left[-p_{j} n I_{j}(x)\right] d x \\
\approx \quad & \exp \left[-n \inf _{x}\left(p_{1} I_{1}(x)+p_{j} I_{j}(x)\right)\right] .
\end{aligned}
$$

Now we show this rigorously. For $n=1,2, \ldots$, set

$$
Z_{n}=\left(\bar{X}_{1}\left(p_{1} n\right), \bar{X}_{j}\left(p_{j} n\right)\right) .
$$

Denote the logarithmic moment generating function of $Z_{n}$ by $\Lambda_{n}\left(\lambda_{1}, \lambda_{j}\right)=\log E\left(e^{\lambda_{1} \bar{X}_{1}\left(p_{1} n\right)+\lambda_{j} \bar{X}_{j}\left(p_{j} n\right)}\right)$ for $\left(\lambda_{1}, \lambda_{j}\right) \in$ $\mathfrak{R}^{2}$.

Lemma 1 The rate function of $\left(Z_{n}: n \geq 0\right)$, $I\left(x_{1}, x_{j}\right)$, equals

$$
p_{1} I_{1}\left(x_{1}\right)+p_{j} I_{j}\left(x_{j}\right) .
$$

Proof It is easily checked that

$$
\frac{1}{n} \Lambda_{n}\left(n \lambda_{1}, n \lambda_{j}\right)=p_{1} \Lambda_{1}\left(\lambda_{1} / p_{1}\right)+p_{j} \Lambda_{j}\left(\lambda_{j} / p_{j}\right),
$$

for $n \geq 1$. Then, by Gartner-Ellis Theorem, $I\left(x_{1}, x_{j}\right)$ equals

$$
\begin{gathered}
\sup _{\lambda_{1}, \lambda_{j}}\left(\lambda_{1} x_{1}+\lambda_{j} x_{j}-p_{1} \Lambda_{1}\left(\lambda_{1} / p_{1}\right)-p_{j} \Lambda_{j}\left(\lambda_{j} / p_{j}\right)\right) \\
=\sup _{\lambda_{1}}\left(\lambda_{1} x_{1}-p_{1} \Lambda_{1}\left(\lambda_{1} / p_{1}\right)\right)+\sup _{\lambda_{j}}\left(\lambda_{j} x_{j}-p_{j} \Lambda_{j}\left(\lambda_{j} / p_{j}\right)\right) .
\end{gathered}
$$

This can be seen to equal

$$
\begin{aligned}
& p_{1} \sup _{\lambda_{1} / p_{1}}\left(\left(\lambda_{1} / p_{1}\right) x_{1}-\Lambda_{1}\left(\lambda_{1} / p_{1}\right)\right)+ \\
& p_{j} \sup _{\lambda_{j} / p_{j}}\left(\left(\lambda_{j} / p_{j}\right) x_{j}-\Lambda_{j}\left(\lambda_{j} / p_{j}\right)\right),
\end{aligned}
$$

and the result follows.
Hence, for $B \in \mathbb{R}^{2}$, such that $\inf _{x \in B^{\circ}} I(x)=$ $\inf _{x \in \bar{B}} I(x)\left(=I_{B}\right)$, we get

$$
\lim _{n \rightarrow \infty} \frac{1}{n} \log P\left(Z_{n} \subset B\right)=-I_{B} .
$$

Therefore,

$$
G_{j}\left(p_{1}, p_{j}\right)=\inf _{x_{1} \geq x_{j}}\left(p_{1} I_{1}\left(x_{1}\right)+p_{j} I_{j}\left(x_{j}\right)\right) .
$$

Since both $I_{1}(x)$ and $I_{j}(x)$ are decreasing in $x$ for $x<\mu_{1}$ and increasing in $x$ for $x>\mu_{j}$, it suffices to search for the infimum for $\mu_{1} \leq x_{j} \leq x_{1} \leq \mu_{j}$. In this region, $I_{1}(x)$ is increasing and $I_{j}(x)$ is decreasing with $x$, so

$$
\begin{aligned}
G_{j}\left(p_{1}, p_{j}\right) & =\inf _{x \in\left[\mu_{1}, \mu_{j}\right]}\left(p_{1} I_{1}(x)+p_{j} I_{j}(x)\right), \\
& =\inf _{x}\left(p_{1} I_{1}(x)+p_{j} I_{j}(x)\right) .
\end{aligned}
$$

For any function $f(x)$, let $f^{\prime}(x)$ and $f^{\prime \prime}(x)$ denote its single and double derivative, respectively, with respect to $x$. Note that for each $x, p_{1} I_{1}(x)+p_{j} I_{j}(x)$ is a concave function of $\left(p_{1}, p_{j}\right)$. Since $G_{j}\left(p_{1}, p_{j}\right)$ is an infimum of concave functions, it is also concave. Also, for $\left(p_{1}, p_{j}\right)>0$, let $x\left(p_{1}, p_{j}\right)$ be the unique solution to

$$
p_{1} I_{1}^{\prime}(x)+p_{j} I_{j}^{\prime}(x)=0 .
$$

It is easily seen that this solution exists and is unique since $I_{1}^{\prime \prime}(x)$ and $I_{j}^{\prime \prime}(x)$ are strictly positive for $x \in\left[\mu_{1}, \mu_{j}\right]$ (as $I_{1}(x)$ and $I_{j}(x)$ are strictly convex in this region), $I_{1}^{\prime}\left(\mu_{1}\right)=0, I_{j}^{\prime}\left(\mu_{1}\right)<0, I_{1}^{\prime}\left(\mu_{j}\right)>0$ and $I_{j}^{\prime}\left(\mu_{j}\right)=0$.

Thus,

$$
G_{j}\left(p_{1}, p_{j}\right)=p_{1} I_{1}\left(x\left(p_{1}, p_{j}\right)\right)+p_{j} I_{j}\left(x\left(p_{1}, p_{j}\right)\right) .
$$

From this and (4) it is easily seen that $\frac{\partial G_{j}\left(p_{1}, p_{j}\right)}{\partial p_{1}}$ equals

$$
\begin{gathered}
I_{1}\left(x\left(p_{1}, p_{j}\right)\right)+p_{1} I_{1}^{\prime}\left(x\left(p_{1}, p_{j}\right)\right) \frac{\partial x\left(p_{1}, p_{j}\right)}{\partial p_{1}} \\
+p_{2} I_{2}^{\prime}\left(x\left(p_{1}, p_{j}\right)\right) \frac{\partial x\left(p_{1}, p_{j}\right)}{\partial p_{1}}=I_{1}\left(x\left(p_{1}, p_{j}\right)\right) .
\end{gathered}
$$

Similarly, $\frac{\partial G_{j}\left(p_{1}, p_{j}\right)}{\partial p_{j}}=I_{j}\left(x\left(p_{1}, p_{j}\right)\right)$. In particular $G_{j}\left(p_{1}, p_{j}\right)$ is strictly increasing in $p_{1}$ and $p_{j}$ for $\left(p_{1}, p_{j}\right)>$ 0 . Also note that $G_{j}\left(p_{1}, p_{j}\right)=0$ if $\min \left(p_{1}, p_{j}\right)=0$.

\section{OPTIMAL ALLOCATION STRATEGY}

Let $p=\left(p_{1}, \ldots, p_{d}\right)$ and $h(p)=\min _{2 \leq j \leq d} G_{j}\left(p_{1}, p_{j}\right)$. Note that $h(\cdot)$, being a minimum of concave functions, is 
concave for $p \geq 0$. From (1) it follows that the problem of determining the allocation that asymptotically minimizes the probability of false selection reduces to solving the concave programming problem: $\max h(p)$ subject to $p \geq 0$ and $\sum_{i=1}^{d} p_{i}=1$.

This may be re-expressed as:

$$
\begin{aligned}
\max & z \quad \text { s.t. } \\
G_{j}\left(p_{1}, p_{j}\right)-z & \geq 0, \quad(2 \leq j \leq d), \\
\sum_{i=1}^{d} p_{i} & =1 \\
p_{j} & \geq 0(2 \leq j \leq d) .
\end{aligned}
$$

Call this optimization problem $\mathbf{P}$. It is well known that the first order conditions are sufficient for global optimality of a concave programming problem (see any standard nonlinear programming text, e.g., Avriel 1976). In the following theorem we use this to determine the optimal allocation strategy.

Theorem 1 Under Assumption 1, if the allocation $p^{*}>0, \sum_{i=1}^{d} p_{i}^{*}=1$, minimizes the asymptotic probability of false selection, then,

$$
\begin{aligned}
\sum_{j=2}^{d} \frac{\partial G_{j}\left(p_{1}^{*}, p_{j}^{*}\right) / \partial p_{1}}{\partial G_{j}\left(p_{1}^{*}, p_{j}^{*}\right) / \partial p_{j}} & =1 \\
G_{j}\left(p_{1}^{*}, p_{j}^{*}\right) & =G_{k}\left(p_{1}^{*}, p_{k}^{*}\right),
\end{aligned}
$$

for $j, k=2, \ldots, d$.

Proof Note that each $p_{i}^{*}$ is strictly positive. This follows as otherwise $h\left(p^{*}\right)=0$ and we know that for $p_{i}=1 / d, i=1, \ldots, d, h(p)>0$. Then from the first order conditions (see, e.g., Avriel 1976), it follows that there exist $\left(\lambda_{j}: j=2, \ldots, d\right)$ and $\gamma$ such that:

$$
\begin{aligned}
1-\sum_{j=2}^{d} \lambda_{j} & =0 \\
\lambda_{j} \frac{\partial G_{j}\left(p_{1}^{*}, p_{j}^{*}\right)}{\partial p_{j}} & =\gamma \quad j=2, \ldots, d \\
\sum_{j=2}^{d} \lambda_{j} \frac{\partial G_{j}\left(p_{1}^{*}, p_{j}^{*}\right)}{\partial p_{1}} & =\gamma \\
\lambda_{j}\left(G_{j}\left(p_{1}^{*}, p_{j}^{*}\right)-z\right) & =0 \quad j=2, \ldots, d .
\end{aligned}
$$

Equation (9) implies that $\lambda_{j}>0$ for some $j$. Since, $\frac{\partial G_{j}\left(p_{1}^{*}, p_{j}^{*}\right)}{\partial p_{j}}>0$ for all $j$, it follows that $\gamma>0$ and each $\lambda_{j}>0$. Then, Equation (8) follows from Equation (12). Equation (7) follows by appropriate substitutions in Equation (11).
Example 1 Consider the case where each $X_{j}$ has a Gaussian distribution with mean $\mu_{j}$ and variance $\sigma_{j}^{2}$. Then it is well known and easily seen that

$$
I_{j}(x)=\frac{\left(x-\mu_{j}\right)^{2}}{2 \sigma_{j}^{2}} .
$$

Now

$$
G_{j}\left(p_{1}, p_{j}\right)=\inf _{x}\left[p_{1} I_{1}(x)+p_{j} I_{j}(x)\right] .
$$

Through differentiation it can be seen that the infimum above is achieved at

$$
x_{j}^{*}=\left(\frac{p_{1} / \sigma_{1}^{2}}{p_{1} / \sigma_{1}^{2}+p_{j} / \sigma_{j}^{2}}\right) \mu_{1}+\left(\frac{p_{j} / \sigma_{j}^{2}}{p_{1} / \sigma_{1}^{2}+p_{j} / \sigma_{j}^{2}}\right) \mu_{j}
$$

and

$$
G_{j}\left(p_{1}, p_{j}\right)=\frac{\left(\mu_{j}-\mu_{1}\right)^{2}}{2\left(\sigma_{1}^{2} / p_{1}+\sigma_{j}^{2} / p_{j}\right)} .
$$

Then,

$$
\frac{\partial G_{j}\left(p_{1}, p_{j}\right)}{\partial p_{1}}=\frac{\left(\mu_{j}-\mu_{1}\right)^{2}}{2\left(\sigma_{1}^{2} / p_{1}+\sigma_{j}^{2} / p_{j}\right)^{2}} \sigma_{1}^{2} / p_{1}^{2} .
$$

Thus, at optimal point for $j, k \geq 2$ :

$$
\frac{\left(\mu_{j}-\mu_{1}\right)^{2}}{\left(\sigma_{1}^{2} / p_{1}^{*}+\sigma_{j}^{2} / p_{j}^{*}\right)}=\frac{\left(\mu_{k}-\mu_{1}\right)^{2}}{\left(\sigma_{1}^{2} / p_{1}^{*}+\sigma_{k}^{2} / p_{k}^{*}\right)},
$$

and

$$
\frac{\partial G_{j}\left(p_{1}^{*}, p_{j}^{*}\right) / \partial p_{1}}{\partial G_{j}\left(p_{1}^{*}, p_{j}^{*}\right) / \partial p_{j}}=\frac{\sigma_{1}^{2} / p_{1}^{* 2}}{\sigma_{j}^{2} / p_{j}^{* 2}},
$$

so that

$$
p_{1}^{*}=\sigma_{1} \sqrt{\sum_{j \geq 2} p_{j}^{* 2} / \sigma_{j}^{2}} .
$$

If, as in Chen et al. (2000), we suppose that $p_{1}^{*}>>p_{j}^{*}$ for $j \geq 2$, then (13) yields

$$
\frac{p_{j}^{*}}{p_{k}^{*}} \approx \frac{\sigma_{j}^{2} /\left(\mu_{j}-\mu_{1}\right)^{2}}{\sigma_{k}^{2} /\left(\mu_{k}-\mu_{1}\right)^{2}} .
$$

This corresponds to the solution proposed by Chen et al. (2000).

Example 2 Consider the case where each $X_{j}$ has a Bernoulli distribution with parameter $q_{j}$. Then it is well 
known and easily seen that

$$
I_{j}(x)=x \log \frac{x}{q_{j}}+(1-x) \log \frac{1-x}{1-q_{j}} .
$$

Again, $G_{j}\left(p_{1}, p_{j}\right)=\inf _{x}\left[p_{1} I_{1}(x)+p_{j} I_{j}(x)\right]$ and it can be seen that the infimum is achieved at

$$
x_{j}^{*}=\frac{\left(\frac{q_{1}}{1-q_{1}}\right)^{p_{1} /\left(p_{1}+p_{j}\right)}\left(\frac{q_{j}}{1-q_{j}}\right)^{p_{j} /\left(p_{1}+p_{j}\right)}}{1+\left(\frac{q_{1}}{1-q_{1}}\right)^{p_{1} /\left(p_{1}+p_{j}\right)}\left(\frac{q_{j}}{1-q_{j}}\right)^{p_{j} /\left(p_{1}+p_{j}\right)}}
$$

and $G_{j}\left(p_{1}, p_{j}\right)$ equals

$$
-\left(p_{1}+p_{j}\right) \log \left[\left(1-q_{1}\right)^{\frac{p_{1}}{p_{1}+p_{j}}}\left(1-q_{j}\right)^{\frac{p_{j}}{p_{1}+p_{j}}}+q_{1}^{\frac{p_{1}}{p_{1}+p_{j}}} q_{j}^{\frac{p_{j}}{p_{1}+p_{j}}}\right] .
$$

We numerically solve for optimal allocation in two cases each with $d=3$. In the first case $q_{1}=0.92$ and $q_{2}=q_{3}=0.99$. In this case $p_{1}^{*}=0.49$ and $p_{2}^{*}=p_{3}^{*}=0.255$. If instead, each $X_{j}$ were assumed to be Gaussian in this case with mean $q_{j}$ and variance $q_{j}\left(1-q_{j}\right)$, then the optimal allocation equals $p_{1}^{*}=0.66$ and $p_{2}^{*}=p_{3}^{*}=0.17$.

In the second case we select $q_{1}=0.5$ and $q_{2}=q_{3}=$ 0.6 , and find that $p_{1}^{*}=0.414$ and $p_{2}^{*}=p_{3}^{*}=0.293$. Again, under the Gaussian assumption, the optimal allocation equals $p_{1}^{*}=0.415$ and $p_{2}^{*}=p_{3}^{*}=0.293$. This suggests that more skewed the distributions are, more misleading the Gaussian assumption may be.

It is important to note that if, instead of each $X_{j}$, we consider an average of batch of $m$ such independent random variables, the log moment generating function of the batch evaluated at $\theta$ equals $m \Lambda_{j}(\theta / m)$ and hence its rate function evaluated at $x$ equals $m I_{j}(x)$. Since all the rate functions are scaled by the same amount $m$, it can be seen that the optimal allocations remain unaffected by this batching operation. In particular, the assumption that for large $m$, the batches have a Gaussian distribution, may lead to misleading allocations.

\section{STOPPING RULE BASED ON INDIFFERENCE ZONE FORMULATION}

We now illustrate how a stopping rule may be developed that provides a conservative upper bound on the probability of false selection in the simple setting where all populations have a Bernoulli distribution. This rule is particularly useful as it exploits the exponential decay rate of the probability of incorrect selection.

Proposition 1 Suppose that each $X_{j}$ is Bernoulli with parameter $q_{j}$ and that $q_{j} \geq q_{1}+\delta$ for $j \geq 2$ and $\delta>0$. Then, under optimal allocation as well as under equal allocation $\left(p_{j}=1 / d\right.$ for all $\left.j\right)$

$$
P(F S) \leq \epsilon
$$

for

$$
n \geq \frac{(\log \epsilon-\log (d-1)) d}{\log \left(1-\delta^{2}\right)} .
$$

Note that $n$ increases very slowly as $\epsilon \rightarrow 0$. The proposition follows from Lemmas 2 and 3.

Lemma 2 Under Assumption 1,

$$
P(F S) \leq(d-1) \exp \left[-n \min _{2 \leq j \leq d} G_{j}(1 / d, 1 / d)\right] .
$$

Lemma 3 Suppose that $q_{j} \geq q_{1}+\delta$ for $j \geq 2$ and $\delta>0$. Then,

$$
\min _{j \geq 2} G_{j}(1 / d, 1 / d) \geq-\frac{1}{d} \log \left(1-\delta^{2}\right) .
$$

Proof of Lemma 2 First we show that

$$
P\left(\bar{X}_{1}\left(p_{1}^{*} n\right) \geq \bar{X}_{j}\left(p_{j}^{*} n\right)\right) \leq \exp \left(-n G_{j}\left(p_{1}^{*}, p_{j}^{*}\right)\right) .
$$

To see this, note that for $\theta \geq 0, P\left(\bar{X}_{1}\left(p_{1}^{*} n\right) \geq \bar{X}_{j}\left(p_{j}^{*} n\right)\right)$ is less than or equal to

$$
E\left(\exp \left[n \theta\left(\bar{X}_{1}\left(p_{1}^{*} n\right)-\bar{X}_{j}\left(p_{j}^{*} n\right)\right)\right] I\left(\bar{X}_{1}\left(p_{1}^{*} n\right) \geq \bar{X}_{j}\left(p_{j}^{*} n\right)\right)\right)
$$

where $I(\cdot)$ above denotes the indicator function. This in turn is

$$
\begin{aligned}
& \leq E\left(\exp \left[n \theta\left(\bar{X}_{1}\left(p_{1}^{*} n\right)-\bar{X}_{j}\left(p_{j}^{*} n\right)\right)\right]\right) \\
& =\exp \left[n p_{1}^{*} \Lambda_{1}\left(\theta / p_{1}^{*}\right)+n p_{j}^{*} \Lambda_{j}\left(-\theta / p_{j}^{*}\right)\right]
\end{aligned}
$$

The last relation holds with $\theta$ replaced by $\theta^{*}>0$ that uniquely satisfies the equation

$$
\Lambda_{1}^{\prime}\left(\theta^{*} / p_{1}^{*}\right)=\Lambda_{j}^{\prime}\left(-\theta^{*} / p_{j}^{*}\right)
$$

To see this, note that $\Lambda_{1}^{\prime}\left(\theta / p_{1}^{*}\right)-\Lambda_{j}^{\prime}\left(-\theta / p_{j}^{*}\right)$ is a strictly increasing continuous function of $\theta$. It equals $\mu_{1}-\mu_{j}<0$ at $\theta=0$ and, under Assumption 1, becomes positive for $\theta$ sufficiently large.

We now argue that

$$
G_{j}\left(p_{1}^{*}, p_{j}^{*}\right)=-p_{1}^{*} \Lambda_{1}\left(\theta^{*} / p_{1}^{*}\right)-p_{j}^{*} \Lambda_{j}\left(-\theta^{*} / p_{j}^{*}\right) .
$$

As argued in (5),

$$
G_{j}\left(p_{1}^{*}, p_{j}^{*}\right)=p_{1}^{*} I_{1}\left(x^{*}\right)+p_{j}^{*} I_{j}\left(x^{*}\right)
$$


where $x^{*}=x\left(p_{1}^{*}, p_{j}^{*}\right)$ satisfies the equation $p_{1}^{*} I_{1}^{\prime}\left(x^{*}\right)=$ $-p_{j}^{*} I_{j}^{\prime}\left(x^{*}\right)$. Set $\theta^{*}$ equal to these two terms. Also note that

$$
I_{1}\left(x^{*}\right)=\theta_{1}\left(x^{*}\right) x^{*}-\Lambda_{1}\left(\theta_{1}\left(x^{*}\right)\right)
$$

where $\theta_{1}\left(x^{*}\right)$ is a unique solution to

$$
\Lambda_{1}^{\prime}(\theta)=x^{*} .
$$

Note that

$I_{1}^{\prime}\left(x^{*}\right)=\theta_{1}\left(x^{*}\right)+\theta_{1}^{\prime}\left(x^{*}\right) x^{*}-\Lambda_{1}^{\prime}\left(\theta_{1}\left(x^{*}\right)\right) \theta_{1}^{\prime}\left(x^{*}\right)=\theta_{1}\left(x^{*}\right)$.

Hence, $\theta_{1}\left(x^{*}\right)=\theta^{*} / p_{1}^{*}$. Similarly,

$$
I_{j}\left(x^{*}\right)=\theta_{j}\left(x^{*}\right) x^{*}-\Lambda_{j}\left(\theta_{j}\left(x^{*}\right)\right)
$$

where $\Lambda_{j}^{\prime}\left(\theta_{j}\left(x^{*}\right)\right)=x^{*}$ so that $I_{j}^{\prime}\left(x^{*}\right)=\theta_{j}\left(x^{*}\right)$, or $\theta_{j}\left(x^{*}\right)=-\theta^{*} / p_{j}^{*}$. From (17), (18) and (19), (16) follows. In particular, we then have

$$
\begin{aligned}
P(F S) & \leq(d-1) \exp \left[-n \min _{2 \leq j \leq d} G_{j}\left(p_{1}^{*}, p_{j}^{*}\right)\right] \\
& \leq(d-1) \exp \left[-n \min _{2 \leq j \leq d} G_{j}(1 / d, 1 / d)\right] .
\end{aligned}
$$

Proof of Lemma 3 Note from Example 2 that

$$
G_{j}(1 / d, 1 / d)=-\frac{2}{d} \log \left[\sqrt{\left(1-q_{1}\right)\left(1-q_{j}\right)}+\sqrt{q_{1} q_{j}}\right] .
$$

It can be easily seen, e.g., through differentiation that $\sqrt{\left(1-q_{1}\right)\left(1-q_{j}\right)}+\sqrt{q_{1} q_{j}}$ is a decreasing function of $q_{j}$ for all $q_{j} \geq q_{1}$. Therefore,

$$
G_{j}(1 / d, 1 / d) \geq-\frac{2}{d} \log \left[\sqrt{\left(1-q_{1}\right)\left(1-q_{1}-\delta\right)}+\sqrt{q_{1}\left(q_{1}+\delta\right)}\right] .
$$

Again through differentiation and some algebraic manipulations it may be seen that $\sqrt{\left(1-q_{1}\right)\left(1-q_{1}-\delta\right)}+$ $\sqrt{q_{1}\left(q_{1}+\delta\right)}$ is maximized at $q_{1}^{*}=(1-\delta) / 2$. From this it follows that

$$
G_{j}(1 / d, 1 / d) \geq-\frac{1}{d} \log \left(1-\delta^{2}\right) .
$$

\section{CONSISTENCY OF OPTIMAL ALLOCATION ESTIMATOR}

In practice, the large deviations rate function corresponding to the probability of false selection is estimated from the generated samples. From implementation viewpoint an adaptive procedure may be developed where a certain number of samples are generated from each population that are then used to estimate optimal allocations. Using these allocations more samples are generated. Combining the generated samples, these allocations are re-estimated and so on.

Note that there may be significant degradation in performance due to estimation errors. This may be considerable if the sample mean observed at any stage of the adaptive procedure does not correspond to the best population, so that the resulting estimated optimal allocations may be very different from their true values. We now argue that as long each population is assigned at least a positive fraction of the total computational budget $n$, the estimated optimal allocations converge to the correct values as $n \rightarrow \infty$. To keep the notation simple, we show this consistency of the estimators when each population is assigned $n$ samples and $n \rightarrow \infty$.

Let $\left(X_{i 1}, \ldots, X_{i n}\right)$ denote the samples generated from population $i$. Suppose that $\Lambda_{i}^{(n)}(\cdot)$ denotes the empirical estimate of the log moment generating function corresponding to population $i$, i.e.,

$$
\Lambda_{i}^{(n)}(\theta)=\log \frac{\sum_{j=1}^{n} \exp \left(\theta X_{i j}\right)}{n},
$$

and

$$
\Lambda^{(n)^{\prime}}(\theta)=\frac{\sum_{j=1}^{n} X_{i j} \exp \left(\theta X_{i j}\right)}{\sum_{i=1}^{n} \exp \left(\theta X_{i j}\right)},
$$

denotes the empirical estimate of the derivative of the $\log$ moment generating function $\Lambda_{i}^{\prime}(\theta)$.

Let

$$
I_{i}^{(n)}(x)=\sup _{\theta}\left(\theta x-\Lambda_{i}^{(n)}(\theta)\right)
$$

denote the estimated rate function from the population $i \leq d$.

Let $\tilde{i}=\arg \min _{i \leq d} \bar{X}_{i}(n)$. Define

$$
G_{j}^{(n)}\left(p_{\tilde{i}}^{\tilde{r}}, p_{j}\right)=\inf _{x}\left(p_{i}^{\sim} I_{\tilde{i}}^{(n)}(x)+p_{j} I_{j}^{(n)}(x)\right)
$$

for $j \neq \tilde{i}$.

Define

$$
h^{(n)}(p)=\min _{j \neq \tilde{i}} G_{j}^{(n)}\left(p_{\tilde{i}}, p_{j}\right) .
$$

Let $p^{(n)} \in \mathfrak{R}^{d}$ denote a solution to the optimization problem: $\max h^{(n)}(p)$ subject to $p \geq 0$ and $\sum_{i=1}^{d} p_{i}=1$. We now state the main result of this section.

Theorem 2 Suppose that Assumption 1 holds and $p^{*}$ uniquely solves $\mathbf{P}$. Then, the empirical estimate of the allocations is consistent, i.e.,

$$
p^{(n)} \rightarrow p^{*} \text { a.s. }
$$

as $n \rightarrow \infty$. 
The proof of this theorem is outlined below. Note that $\tilde{i} \rightarrow 1$ as $n \rightarrow \infty$ a.s. In view of this, we set

$$
G_{j}^{(n)}\left(p_{\tilde{i}}, p_{j}\right)=G_{j}^{(n)}\left(p_{1}, p_{j}\right),
$$

to simplify the proofs.

\subsection{Proof Outline of Consistency}

Let $\bar{\theta}_{j}$ denote the solution to $\Lambda_{j}^{\prime}(\theta)=\mu_{d}$ and $\underline{\theta}_{j}$ denote the solution to $\Lambda_{j}^{\prime}(\theta)=\mu_{1}$. Due to Assumption 1, these exist and are finite. For notational convenience we drop the subscript $j$ until necessary. Lemmas 4, 5, 6 and 7 are useful in the proof of Theorem 2.

Lemma 4 Under Assumption 1, $\Lambda^{(n)}(\theta) \rightarrow \Lambda(\theta)$ and $\Lambda^{(n)^{\prime}}(\theta) \rightarrow \Lambda^{\prime}(\theta)$ for all $\theta \in[\underline{\theta}, \bar{\theta}]$ a.s.

Proof outline Note that $\Lambda^{(n)}(\theta) \rightarrow \Lambda(\theta)$ and $\Lambda^{(n)^{\prime}}(\theta) \rightarrow \Lambda^{\prime}(\theta)$ a.s. for each $\theta \in[\underline{\theta}, \bar{\theta}]$. Hence, this convergence holds for all rational $\theta \in[\underline{\theta}, \bar{\theta}]$ and $\bar{\theta}$ along a set of paths of probability 1 . Call this set $A$. It is easy to see that along $A$, this convergence holds for all $\theta \in[\underline{\theta}, \bar{\theta}]$. To see this, note that for any irrational $\theta$,

$$
\left|\Lambda^{(n)}(\theta)-\Lambda(\theta)\right|
$$

is upper bounded by

$$
\left|\Lambda^{(n)}(\theta)-\Lambda^{(n)}\left(\theta_{r}\right)\right|+\left|\Lambda^{(n)}\left(\theta_{r}\right)-\Lambda\left(\theta_{r}\right)\right|+\left|\Lambda\left(\theta_{r}\right)-\Lambda(\theta)\right|
$$

where $\theta_{r}$ is a rational number in the neighborhood of $\theta$. Since $\Lambda^{(n)}(\cdot)$ is convex,

$$
\left|\Lambda^{(n)}(\theta)-\Lambda^{(n)}\left(\theta_{r}\right)\right| \leq \Lambda^{(n)^{\prime}}(\bar{\theta})\left|\theta-\theta_{r}\right|
$$

Now, $\Lambda^{(n)^{\prime}}(\bar{\theta}) \rightarrow \Lambda^{\prime}(\bar{\theta})$ and thus is bounded for $n$ sufficiently large (this bound may depend on the sample path). Hence, it follows that by choosing $\theta_{r}$ sufficiently close to $\theta$, and $n$ sufficiently large, (20) can be made arbitrarily small. Thus, $\Lambda^{(n)}(\theta) \rightarrow \Lambda(\theta)$ along $A$ for all $\theta \in[\underline{\theta}, \bar{\theta}]$.

Lemma 5 Under Assumption 1,

$$
I^{(n)}(x) \rightarrow I(x)
$$

a.s.

To see this, let $\theta^{*}$ denote the solution to

$$
\Lambda^{\prime}(\theta)=x
$$

Then,

$$
I(x)=\theta^{*} x-\Lambda\left(\theta^{*}\right) .
$$

As argued in the proof of Lemma 2,

$$
I^{\prime}(x)=\theta^{*}
$$

Let $\theta^{(n)}$ denote the solution to

$$
\Lambda^{(n)^{\prime}}(\theta)=x
$$

whenever it exists.

Proof outline Note that for any $\epsilon>0 \Lambda^{(n)^{\prime}}\left(\theta^{*}-\epsilon\right) \rightarrow$ $\Lambda^{\prime}\left(\theta^{*}-\epsilon\right)<x$ and $\Lambda^{(n)^{\prime}}\left(\theta^{*}+\epsilon\right) \rightarrow \Lambda^{\prime}\left(\theta^{*}+\epsilon\right)>x$. It follows that for $n$ sufficiently large, $\theta^{(n)}$ exists. Furthermore, $\theta^{(n)} \in\left(\theta^{*} \pm \epsilon\right)$ for all $n$ sufficiently large and since $\epsilon$ is arbitrary, $\theta^{(n)} \rightarrow \theta^{*}$ a.s. Then,

$$
I^{(n)}(x)=\theta^{(n)} x-\Lambda^{(n)}\left(\theta^{(n)}\right),
$$

for all $n$ sufficiently large. To prove the result we need to show that

$$
\Lambda^{(n)}\left(\theta^{(n)}\right) \rightarrow \Lambda\left(\theta^{*}\right)
$$

a.s. Note that $\left|\Lambda^{(n)}\left(\theta^{(n)}\right)-\Lambda\left(\theta^{*}\right)\right|$

$$
\leq\left|\Lambda^{(n)}\left(\theta^{(n)}\right)-\Lambda^{(n)}\left(\theta^{*}\right)\right|+\left|\Lambda^{(n)}\left(\theta^{*}\right)-\Lambda\left(\theta^{*}\right)\right| .
$$

Since the last term goes to zero as $n \rightarrow \infty$, and since

$$
\left|\Lambda^{(n)}\left(\theta^{(n)}\right)-\Lambda^{(n)}\left(\theta^{*}\right)\right| \leq \Lambda^{(n)^{\prime}}(\bar{\theta})\left|\theta^{(n)}-\theta^{*}\right|,
$$

the result follows.

Lemma 6 Under Assumption 1, for $p_{1}, p_{j}>0$,

$$
G_{j}^{(n)}\left(p_{1}, p_{j}\right) \rightarrow G_{j}\left(p_{1}, p_{j}\right)
$$

a.s.

Proof outline From (22), note that $I_{1}^{\prime}(x)=\theta_{1}^{*}$ and from the proof of Lemma 5, it follows that for $n$ sufficiently large, $\theta_{1}^{(n)}$ is well defined and $I_{1}^{(n)^{\prime}}(x)=\theta_{1}^{(n)}$. Thus, from Lemma 5 and its proof it follows that $I_{i}^{(n)}(x) \rightarrow I_{i}(x)$, $I_{i}^{(n)^{\prime}}(x) \rightarrow I_{i}^{\prime}(x)$ a.s. for any $x \in\left[\mu_{1}, \mu_{d}\right]$ and $i \leq d$. It is easy to show that this is true a.s. simultaneously for all $x \in\left[\mu_{1}, \mu_{d}\right]$.

Let $x^{*}$ be the solution to

$$
p_{1} I_{1}^{\prime}(x)+p_{2} I_{j}^{\prime}(x)=0 .
$$

As argued in the proof of Lemma 5, we can show that for sufficiently large $n$, there exists $x^{(n)}$, a solution to

$$
p_{1} I_{1}^{(n)^{\prime}}(x)+p_{2} I_{j}^{(n)^{\prime}}(x)=0,
$$


and that $x^{(n)} \rightarrow x^{*}$. Then, for sufficiently large $n$,

$$
G_{j}^{(n)}\left(p_{1}, p_{j}\right)=p_{1} I_{1}^{(n)}\left(x^{(n)}\right)+p_{2} I_{j}^{(n)}\left(x^{(n)}\right) .
$$

Again, as in the proof of Lemma 5, we can show that $I_{1}^{(n)}\left(x^{(n)}\right) \rightarrow I_{1}\left(x^{*}\right)$ and $I_{j}^{(n)}\left(x^{(n)}\right) \rightarrow I_{j}\left(x^{*}\right)$ a.s. so that the result follows.

It therefore follows that $h^{(n)}(p) \rightarrow h(p)$ a.s.

From the definitions of $x^{*}$ and $x^{(n)}$ specified in the proof of Lemma 6, and (6), it follows that

$$
\frac{\partial}{\partial p_{1}} G_{j}\left(p_{1}, p_{j}\right)=I_{1}\left(x^{*}\right)
$$

and

$$
\frac{\partial}{\partial p_{j}} G_{j}\left(p_{1}, p_{j}\right)=I_{j}\left(x^{*}\right) .
$$

Similarly, $\quad \frac{\partial}{\partial p_{1}} G_{j}^{(n)}\left(p_{1}, p_{j}\right) \quad=\quad I_{1}^{(n)}\left(x^{(n)}\right) \quad$ and $\frac{\partial}{\partial p_{j}} G_{j}^{(n)}\left(p_{1}, p_{j}\right)=I_{j}^{(n)}\left(x^{(n)}\right)$ for $n$ sufficiently large.

If $\left(\hat{p}^{(n)} \geq 0: n \geq 1\right)$ lie in a compact set, it is easy to see that the partial derivatives $\frac{\partial}{\partial p_{1}} G_{j}^{(n)}\left(\hat{p}_{1}^{(n)}, \hat{p}_{j}^{(n)}\right)$ and $\frac{\partial}{\partial p_{j}} G_{j}^{(n)}\left(\hat{p}_{1}^{(n)}, \hat{p}_{j}^{(n)}\right)$ are uniformly bounded for all $n$ sufficiently large, a.s. Then the following lemma follows:

Lemma $7 \quad$ Suppose that $\left(\hat{p}^{(n)} \geq 0: n \geq 1\right)$ lie in a compact set and $\hat{p}^{(n)} \rightarrow \hat{p}$. Then

$$
G_{j}^{(n)}\left(\hat{p}_{1}^{(n)}, \hat{p}_{j}^{(n)}\right) \rightarrow G_{j}\left(\hat{p}_{1}, \hat{p}_{j}\right) .
$$

\section{Proof outline of Theorem 2}

The sequence $\left(p^{(n)}: n \geq 1\right)$ lies in a compact set and hence there exists a convergent subsequence $\left(p^{\left(n_{k}\right)}: k \geq 1\right)$ such that

$$
p^{\left(n_{k}\right)} \rightarrow \tilde{p}
$$

for some $\tilde{p}$ that may be sample path dependent. Note that

$$
h^{\left(n_{k}\right)}\left(p^{\left(n_{k}\right)}\right) \leq h^{\left(n_{k}\right)}(p) .
$$

Thus,

$$
\lim _{k \rightarrow \infty} h^{\left(n_{k}\right)}\left(p^{\left(n_{k}\right)}\right) \leq \lim _{k \rightarrow \infty} h^{\left(n_{k}\right)}(p)=h(p)
$$

for all $p \geq 0, \sum_{i \leq d} p_{i}=1$. Once we show that

$$
\lim _{k \rightarrow \infty} h^{\left(n_{k}\right)}\left(p^{\left(n_{k}\right)}\right)=h(\tilde{p}),
$$

it then follows that

$$
h(\tilde{p}) \leq \inf _{p \geq 0, \sum_{i \leq d} p_{i}=1} h(p)=h\left(p^{*}\right)
$$

so that $\tilde{p}=p^{*}$ a.s. Since every subsequence of $\left(p^{(n)}: n \geq\right.$ 1) will have a further subsequence that converges to $p^{*}$, the result then follows. We now argue that (24) holds. To see this, note that

$\left|h^{\left(n_{k}\right)}\left(p^{\left(n_{k}\right)}\right)-h(\tilde{p})\right| \leq \sum_{2 \leq j \leq d}\left|G_{j}^{\left(n_{k}\right)}\left(p_{1}^{\left(n_{k}\right)}, p_{j}^{\left(n_{k}\right)}\right)-G_{j}\left(\tilde{p}_{1}, \tilde{p}_{j}\right)\right|$

and (24) follows from (23).

\section{REFERENCES}

Avriel, M. 1976. Nonlinear Programming: Analysis and Methods. Prentice- Hall.

Chen, C.H., J. Lin, E. Yucesan and S. E. Chick. 2000. Simulation Budget Allocation for Further Enhancing the Efficiency of Ordinal Optimization. Journal of Discrete Event Dynamic Systems: Theory and Applications, Vol. 10, 251-270.

Dai, L. 1996. Convergence Properties of Ordinal Comparison in the Simulation of Discrete Event Dynamic Systems. Journal of Optimization Theory and Applications, Vol. 91, 2, 363-388.

Dembo, A., and O. Zeitouni. 1992. Large Deviations Techniques and Applications. Jones and Bartlett, Boston, MA.

Goldsman, D., and B. L. Nelson. 2001. Statistical Selection of Best System. Proceedings of the 2001 Winter Simulation Conference, Ed. B. A. Peters, J. S. Smith, D. J. Medeiros, and M. W. Rohrer. 139-146. Piscataway, New Jersey: Institute of Electrical and Electronics Engineers.

Kim, S. H., and B. L. Nelson. 2003. Selecting the Best System: Theory and Methods. Proceedings of the 2003 Winter Simulation Conference, Ed. S. Chick, P. J. Sanchez, D. Ferrin, and D. J. Morrice. 101-112. Piscataway, New Jersey: Institute of Electrical and Electronics Engineers.

\section{AUTHOR BIOGRAPHIES}

PETER W. GLYNN received his Ph. D. from Stanford University, after which he joined the faculty of the Department of Industrial Engineering at the University of Wisconsin-Madison. In 1987, he returned to Stanford, where he is the Thomas Ford Professor of Engineering in the Department of Management Science and Engineering. His research interests include discrete-event simulation, computational probability, queueing and gen- 
eral theory for stochastic systems. His e-mail address is $<$ glynn@leland.stanford.edu>.

SANDEEP JUNEJA received his Ph.D. from Stanford University, after which he joined American Credit Indemnity, Baltimore (1993-95) and Andersen Consulting, Delhi (199596). He then joined Indian Institute of Technology Delhi as a faculty (1996-2002). Currently, he is an academic member in the School of Technology and Computer Science in the Tata Institute of Fundamental Research. His research interests include applied probability with a focus on discrete-event simulation of stochastic systems. His email address is $<j u n e j a @ t i f r . r e s . i n>$ and his web page is <www.tcs.tifr.res.in/ ${ }^{\text {sandeepj }}>$ 